\title{
Thermal Unbinding of Highly Oriented Phospholipid Membranes
}

\author{
M. Vogel, ${ }^{1,2}$ C. Münster, ${ }^{1}$ W. Fenzl, ${ }^{2}$ and T. Salditt ${ }^{1}$ \\ ${ }^{1}$ Sektion Physik der Ludwig-Maximilians-Universität München, Geschwister-Scholl-Platz 1, D-80539 München, Germany \\ ${ }^{2}$ Max-Planck-Institut für Kolloid- und Grenzflächenforschung, D-14424 Potsdam, Germany
}

(Received 6 April 1999)

\begin{abstract}
We present a temperature dependent x-ray reflectivity study of highly oriented, fully hydrated multilamellar phospholipid membranes. Both the specular and diffuse (nonspecular) x-ray reflectivity were measured for dimyristoyl-sn-glycero-phosphocholine (DMPC) and oleoyl-palmitoyl-sn-glycero-phosphocholine (POPC) on silicon substrates in excess water. In this configuration the repeat distance as well as the fluctuation spectra can be determined as a function of temperature. Both model systems studied exhibit a discontinuous unbinding transition from a substrate bound, multilamellar state to a state of freely dispersed bilayers in water. In the unbound phase a single membrane remains on the substrate.
\end{abstract}

PACS numbers: 87.16.Dg, 61.10.Kw, 87.15.Ya

Phospholipid bilayers, which are composed of two opposing monolayers of phospholipid molecules, tend to form a lyotropic smectic liquid crystal unless repulsive electrostatic or entropic undulation forces overcome the attractive van der Waals interaction [1]. Under certain well-chosen conditions one expects a continuous transition from a bound to an unbound state of a single bilayer in a given molecular potential, e.g., near a wall [2]. The simple picture valid for single membranes has been extended for multilamellar systems to a much more complicated scenario [3]. This so-called unbinding transition has been extensively investigated theoretically since it was proposed more than ten years ago. However, there is not much experimental evidence for the existence of such a transition in a system of phospholipid bilayers. A possible candidate has been found by Helfrich and Mutz, who interpret observations with DGDG (digalactosyl diglyceride) stacks using optical microscopy as an unbinding transition [4]. Furthermore, pretransitional fluctuations close to the chain melting transition lead to an increase of the repeat distance $d$ as predicted for an unbinding transition and have been observed in different phosphocholine (PC) lipids [5]. However, neither was it possible to observe the transition itself in this case, nor could the effect be unambiguously linked to a swelling of the water layer rather than of the bilayer itself [6].

In contrast to the large body of experimental studies of membrane structure conducted in isotropic solution, we use highly oriented multilamellar stacks of bilayers deposited on silicon substrates [7,8]. Such samples are amenable to powerful, interface sensitive scattering techniques (specular and nonspecular scattering, grazing incidence diffraction) avoiding the ambiguities associated with powder averaging. In this paper we demonstrate that highly oriented systems with a mosaicity of below the instrumental resolution, typically below $0.01^{\circ}$, can be prepared under conditions of full hydration in excess water, and that the unbinding transition can be indeed observed and studied quantitatively in the setup sketched in Fig. 1(a). Specifically, we present results on the two zwitterionic, neutral phospholipid systems dimyristoyl-snglycero-phosphocholine (DMPC) and oleoyl-palmitoylsn-glycero-phosphocholine (POPC).

Following Seul and Sammon [9], the lipids were dissolved in 2-isopropanol in different concentrations, and pipetted onto $14 \times 25 \mathrm{~mm}^{2}$ carefully cleaned (100) silicon substrates. The evaporation rate of the solvent was controlled in a cascade of chambers over a period of $12 \mathrm{~h}$ before keeping the samples in a desiccator for another $24 \mathrm{~h}$ and consecutive rehydration in ultrapure $\mathrm{H}_{2} \mathrm{O}$. After several tempering cycles the procedure yielded samples of an extremely low mosaicity, e.g., $5 \times 10^{-3}$ degrees for POPC, see Fig. 1(b), which was preserved after filling the sample chamber with water and swelling the membranes to their equilibrium values, which are never quite reached in the case of hydration from the water vapor [10]. Typically, an overall film thickness $D$ in between 1 and $10 \mu \mathrm{m}$ was obtained, depending on the amount of lipid used in the initial spreading. The x-ray reflectivity experiments were carried out at the bending magnet beam lines D4 and E2 at Hasylab/DESY in Hamburg and at the undulator beam line ID1 of the ESRF (European Synchrotron Radiation Facility) in Grenoble, using a collimated x-ray beam of $20 \mathrm{keV}$ photon energy set by double bounce Si monochromators,
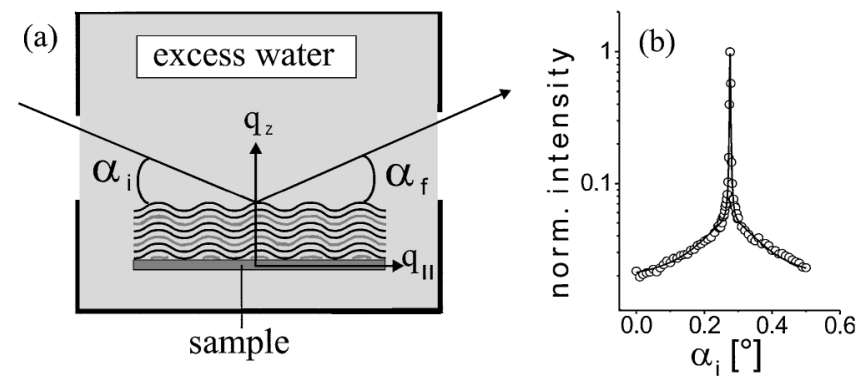

FIG. 1. (a) Setup of the x-ray measurements on fully hydrated, highly oriented phospholipid membranes. (b) Rocking scan at the first Bragg peak of a multilamellar POPC sample with a width (FWHM) of $0.005^{\circ}$. Diffuse scattering is observed about 1.5 orders of magnitude below the specular peak. 
to transverse the $18 \mathrm{~mm}$ of bulk water in the chamber. At the same time the relatively high energy helped to prevent any observable radiation damage.

In Fig. 2 the reflectivity of POPC in millipore (saltfree) water is shown at different temperatures, with the corresponding curves shifted vertically for clarity. The equidistant set of sharp single-domain Bragg peaks reflects the multilamellar periodicity of around $d=d_{w}+d_{m}=$ $64 \AA$, where $d_{m} \simeq 39 \AA$ is the bilayer thickness and $d_{w} \simeq$ $25 \AA$ the water layer, as determined from a Fourier reconstruction of the projected electron density profile. The repeat distance is set by a balance of the attractive van der Waals interactions, and a combination of repulsive hydration and undulation interaction, since POPC and DMPC are charge neutral. The radial FWHM of the peaks is typically below $2 \cdot 10^{-3} \AA^{-1}$, indicating vertical domain sizes of above $1 \mu \mathrm{m}$. At full hydration, $d$ stays almost constant over a temperature range between 10 and $76^{\circ} \mathrm{C}$ shifting monotonically from 62.9 to $64 \AA$. Then, at $80^{\circ} \mathrm{C}$ the stack unbinds discontinuously from the substrate and dissolves, probably in the form of vesicles into the excess water phase. A single adsorbed membrane is left behind up to high temperatures, as proven by the modulations of the Fresnel reflectivity. An analogous scenario has been observed for DMPC with a transition temperature of $T_{u}=95^{\circ} \mathrm{C}$. The analysis of the resulting single membrane is presented in more detail in Fig. 5.

Analytical treatments of the unbinding transition are based on an analogy between strings in two dimensions

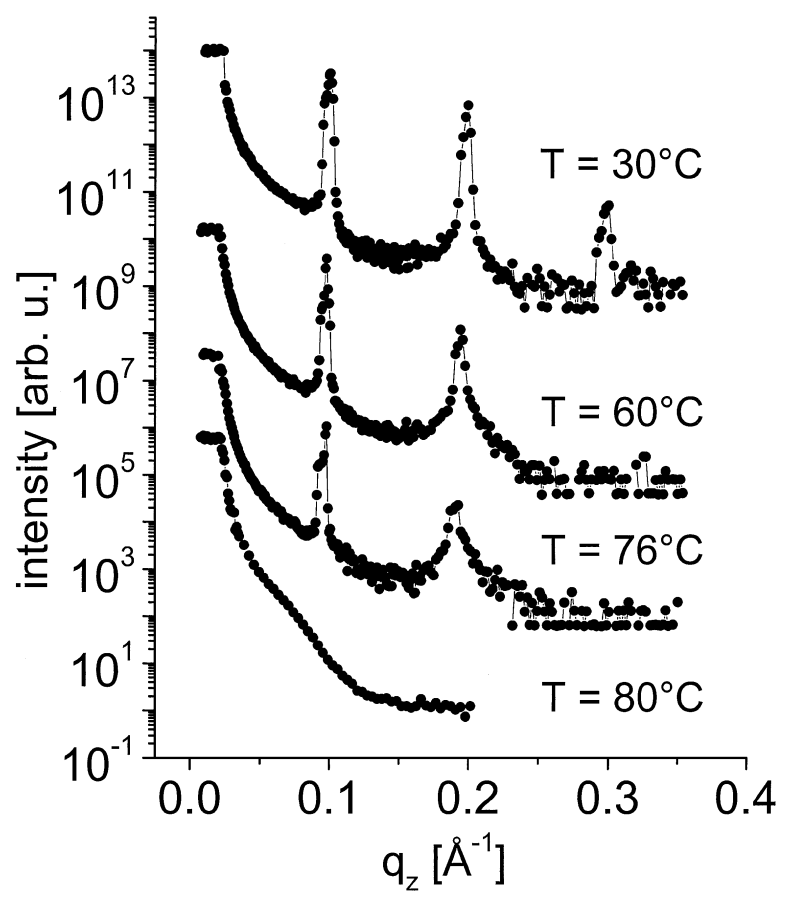

FIG. 2. (a) Reflectivity of multilamellar POPC membranes at different temperatures $T$ (shifted vertically for clarity). The curve at $T=80{ }^{\circ} \mathrm{C}$ corresponds to a single membrane on the substrate. and membranes in three dimensions. A rich scenario of unbinding transitions is predicted depending on the relative strength of the membrane-substrate and membrane-membrane interaction. The bilayer positions $z_{i}$ in the multilamellar stack are predicted to diverge at $T_{u}(i)$ as

$$
z_{i}(T)-z_{i}(T=0) \approx A_{i}(N)\left(\frac{1}{T}-\frac{1}{T_{u}(i)}\right)^{-1},
$$

resulting in a critical swelling of $d$ with $N$-dependent critical amplitudes. For comparison, the thickness of the water layer $d_{w}$ is plotted against $T$ in Fig. 3, together with the corresponding theoretical curve calculated for $N=2$. However, scaling arguments indicate that the critical regime becomes increasingly small in the limit of large $N$, such that it may not be reached in the present experiment of $N \simeq 100$ [3]. Indeed, theory predicts that for large $N$ the unbinding transition will resemble a discontinuous transition.

While $d$ stays almost constant, one can observe a pronounced decrease in the intensities of the Bragg reflections with increasing $T$ in the raw data of Fig. 2, namely of the higher order peaks, but quantitatively different from simple Debye-Waller-like behavior. As a precursor effect to the discontinuous unbinding transition, the intensity decrease of the higher order peaks indicates a change of the lamellar fluctuations and elasticity parameters with $T$. In order to gain an understanding of how the observed changes relate to the molecular interbilayer potential, we have analyzed the bilayer fluctuations on the basis of linear smectic elasticity theory [11]. Pronounced power-law peak line shapes are predicted as a result of diverging positional correlations in an infinitely large system (LandauPeierls instability). Accordingly, the intensity decays as $S\left(q_{z}-q_{z, n}\right) \propto\left(q_{z}-q_{z, n}\right)^{-\left(2-\eta_{n}\right)}$ with $q_{z, n}$ denoting the center of the $n$th order peak. The finite size $D$ in the

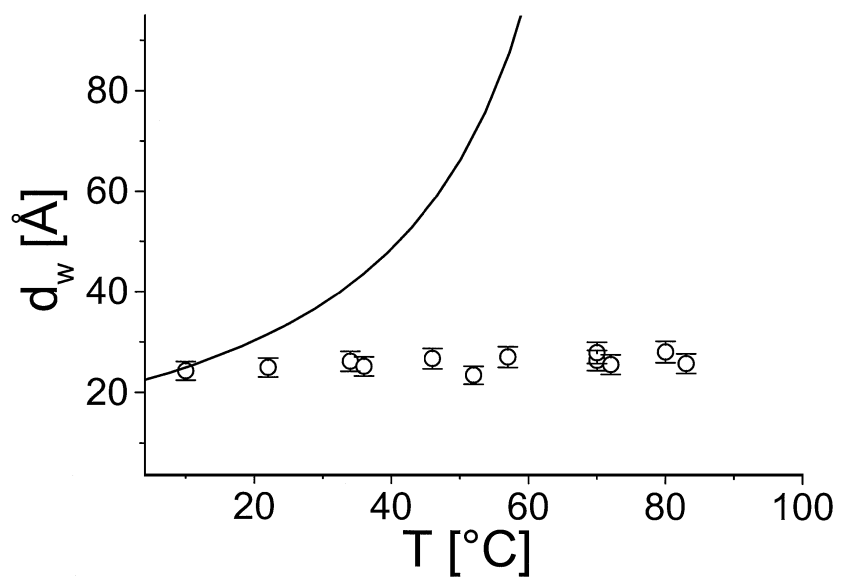

FIG. 3. The thickness of the water layer between membranes $d_{w}$ as a function of $T$, compared to the theoretic prediction of critical unbinding for the case $N=2$ (solid line). Note, however, that the critical regime may decrease strongly with $N$ (Ref. [3]). 
stacking $(z)$ direction removes the singularity at $q_{z, n}$ and leads to a peak line shape which can be described by a superposition of a sharp Bragg-like component and the above power-law singularity [12-15].

The line shape exponent $\eta_{n}$ is defined by $\eta_{n}=$ $n^{2} \pi k_{B} T /(2 d \sqrt{K B})$, where $B\left(\mathrm{~J} / \mathrm{m}^{3}\right)$ and $K(\mathrm{~J} / \mathrm{m})$ are the bulk moduli for compression and curvature. $K$ can be related to the bending modulus of a single membrane according to $K=K_{S} / d$. Typical profiles are shown in Fig. 4(a) for $n=2$ at temperatures $T=33.2^{\circ} \mathrm{C}$ and $T=50.1{ }^{\circ} \mathrm{C}$, respectively, on double logarithmic scale, along with the corresponding fits (solid lines) and the slope of the resolution function (dashed). The characteristic power-law decay is clearly visible. Moreover, a systematic increase of $\eta_{n}$ with order $n$ is observed, e.g., see Fig. 4(b) for POPC at $T=10^{\circ} \mathrm{C}$, where five peaks have been observed. While the general trend is well captured for values of $\eta_{n} / n^{2}=0.07 \pm 0.03$, there are significant deficiencies of the fit. The finite size and the boundary conditions of a flat substrate are not expected to change the line shape exponent $\eta$ [13]. Therefore we attribute the discrepancies to the limitations of the simple, harmonic model possibly resulting from nonlinear effects, lateral tension, and static defects.

The boundary condition of a flat substrate leads to a suppression of the long range lateral fluctuations, leading to a clear separation of specular (Bragg-like) and nonspecular (diffuse) scattering, as illustrated in the angular scan (rocking scan) through the first Bragg peak shown in Fig. 1(b). Analyzing the data in a simple approach by a double-Lorentzian fit (solid line), one can determine the relevant lateral length scales implicit in the data $[16,17]$. The sharp specular peak width (FWHM) of $0.003^{\circ}$ sets a

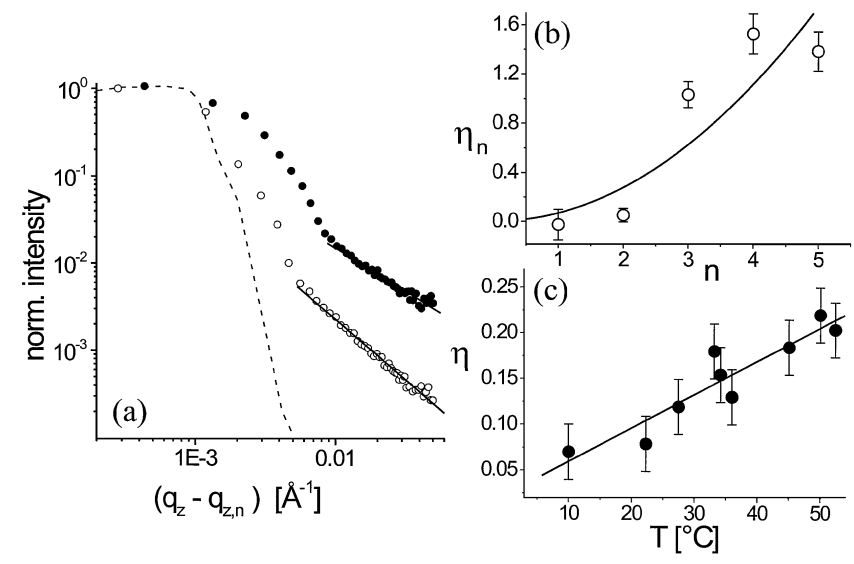

FIG. 4. (a) The second order Bragg peak of POPC at $T=$ $33.2{ }^{\circ} \mathrm{C}$ (open symbols) and $T=50.1^{\circ} \mathrm{C}$ (solid symbols) on double-logarithmic scale as a function of $q_{z}-q_{c}$, along with the resolution function (dashed). (b) The dependence of the line shape parameter $\eta_{n}$ for POPC at $T=10^{\circ} \mathrm{C}$, along with the harmonic Caillé scaling law for $\eta=0.07$. (c) The temperature dependence of the line shape exponent $\eta$, indicating an increase in lamellar disorder starting well below $T_{u}$. lower limit of about $40 \mu \mathrm{m}$ for the lateral expension $L$ of the lamellar domains, while the broad diffuse component indicates the presence of fluctuations on lateral length scales of up to $\xi_{\|} \simeq 0.6 \mu \mathrm{m}$, above which the membranes are essentially flat. Accordingly, wavelengths larger than the lateral correlation length $\xi_{\|}$do not exist, since the corresponding amplitudes cannot decay over the total film thickness $D$.

For smectics the damping in $z$ is given by $\exp \left(-z / \lambda q_{\|}^{2}\right)$ with $\lambda=\sqrt{K / B}$ and $q_{\|}$denoting the wave vector of the corresponding fluctuation, from which we estimate $\lambda \simeq$ $21 \AA$ for $D \simeq 5 \mu \mathrm{m}$ and $T=14{ }^{\circ} \mathrm{C}$. Along with the value of $\eta \simeq 0.07$ determined above, it follows that the single bilayer bending rigidity is on the order of $K_{s} \simeq 7 k_{B} T$ which is somewhat lower than the value of $10 k_{B} T$ published recently for POPC [18]. The estimation of $\lambda$ was confirmed by plotting the $q_{z}$ width of the quasi-Bragg peaks as a function of $q_{\|}$. From the same numbers of $\eta$ and $\lambda$ we also get $B \simeq 0.5 \times 10^{-14} \mathrm{~J} / \mathrm{m}$. The results of the analysis are shown in Fig. 4(c) where the exponent $\eta=\eta_{n} / n^{2}$ is plotted as a function of $T$. Over the range covered in $T, \eta(T)$ can be well approximated by a linear relationship [19], extrapolating to a value of $\eta\left(T_{u}\right)=0.31$ just below the unbinding transition. Since at the same time, $\lambda$ is estimated to increase with $T$ from about $20 \AA$ to about twice that value at $T_{u}$, most of the changes with temperature in between $10^{\circ} \mathrm{C} \leq T \leq T_{u}$ translate into a decrease of $B$ with $B\left(T_{u}\right) \simeq 0.08 B\left(T=10^{\circ} \mathrm{C}\right)$, while the bending rigidity $K$ is varying less strongly, approximately as $K\left(T_{u}\right) \simeq 0.7 K\left(T=10^{\circ} \mathrm{C}\right)$. Therefore, the main effect is a softening of the interbilayer potential as a precursor to the unbinding transition. Note, however, that $B$ does not diverge as would be the case if the transition were continuous.

Let us now turn to the high temperature phase with the lipid membranes dispersed in the excess water and one bilayer remaining at the surface. Obviously, the reflectivity technique is sensitive to the latter only. For quantitative analysis, the measured values of DMPC at $T=95^{\circ} \mathrm{C}$ are first normalized to the Fresnel reflectivity of a sharp interface water/silicon; see data symbols in Fig. 5. The data are then fitted to a box model of a single membrane of DMPC in direct contact with excess water and separated from the substrate by an intermediate water layer. The thickness of the headgroup and tail regions, $8.6 \pm 1 \AA$ and $9.6 \pm 1 \AA$ for a single head group and a single chain, respectively, is in good agreement with published data and does not really represent free parameters. Equally constraint are the densities of water, silicon, and also the membrane. The only distance entering as a free parameter is the water layer thickness $l_{w}$, which is appropriate given the rather limited structure of the dataset. This parameter is determined by a simulation in simple Born approximation [20] to $l_{w}=8.7 \pm 1 \AA$. At the same time, an average root mean square (rms) fluctuation amplitude (roughness) of $10 \AA$ has to be assumed for the membrane. The inset in 


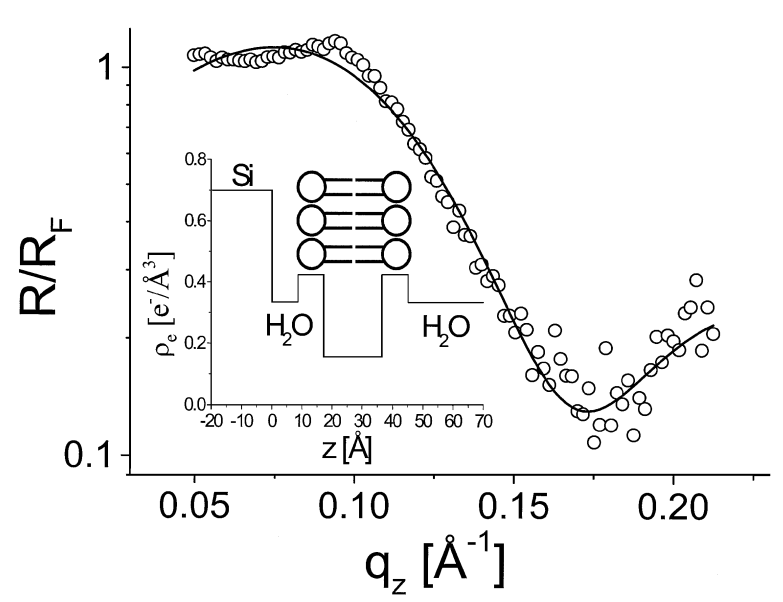

FIG. 5. Reflectivity of a single DMPC membrane on the silicon substrate after the unbinding of the multilamellar DMPC sample (normalized to the Fresnel reflectivity). The solid line is a fit corresponding to the density profile shown in the inset.

Fig. 5 shows the electron density profile corresponding to the fit.

In summary, we are faced with the following experimental findings: Multilamellar membranes of POPC and DMPC unbind from the substrate in a discontinuous transition at a temperature $T_{u}$. Below $T_{u}$ neither a critical swelling nor an increase of lateral fluctuations are observed, indicating that steric repulsion is not driving the transition. Moreover, undulation induced unbinding should be accompanied by an increase in critical diffuse scattering, which was not observed. The conclusion is further supported by the fact that two different lipid membranes, which differ in bending rigidity by more than a factor of 2, unbind at nearly identical absolute temperatures. On the other hand, the transition cannot be trivially explained by a mere dissolution of the bilayers, since the critical micelle concentration at $T_{u}$ is still minute.

The analysis of the multilamellar fluctuations below and at the unbinding transitions yields only partial agreement with the simple harmonic model of smectic elasticity. Extracting quantitative results for $B$ and $K$ by this model, we find that at $T_{u}$ the compressibility decreases by about an order of magnitude while the bending rigidity is lowered by about $30 \%$ (as compared to $T=10^{\circ} \mathrm{C}$ ). This result is hard to reconcile with the observation that $d$ does not increase considerably at $T_{u}$. Therefore we speculate that $B$ may not be determined by the molecular interactions alone, but also by other parameters, in particular by static defects. Accordingly, the temperature dependent properties of lamellar defects, such as defect density and mobility, may determine the stability of the bound phase. Consequently, unbinding may be associated with a change in the defect structure, in a similar manner as defects are responsible for two-dimensional melting. This unbinding scenario may very well be accompanied by the precursor effects observed here. Finally, the setup of highly aligned membranes in excess water put forward in the present study and the associated methods of x-ray scattering can be used to shed light on many current, structural problems in membrane biophysics, including the recently discussed vapor pressure paradoxon [10], anomalous swelling near the main phase transition $[5,6]$, as well as fundamental structural aspects of lipid-peptide and lipid-protein interaction.

We thank C. Safinya, J. Krumhans'l, and R. Netz for fruitful discussions, A. Plech for making his sample chamber available to us, and particularly J. Peisl and R. Lipowsky for their continuous support. We are further grateful for the excellent beam and working conditions provided by the staff of ESRF and HASYLAB.

[1] Structure and Dynamics of Membranes, edited by R. Lipowsky and E. Sackmann, Handbook of Biological Physics (Elsevier, Amsterdam, 1995), Vol. 1, Pts. A \& B.

[2] R. Lipowsky and S. Leibler, Phys. Rev. Lett. 56, 2541 (1986).

[3] R. Netz and R. Lipowsky, Phys. Rev. Lett. 71, 3596 (1993); R. Lipowsky, Z. Phys. B 97, 193 (1995).

[4] M. Mutz and W. Helfrich, Phys. Rev. Lett. 62, 2881 (1989).

[5] J. Lemmich et al., Phys. Rev. Lett. 75, 3958 (1995).

[6] R. Zhang et al., Phys. Rev. Lett. 74, 2832 (1995).

[7] M. Seul and P. Eisenberger, Phys. Rev. A 39, 4230 (1989).

[8] D. C. Wack and W. W. Webb, Phys. Rev. A 40, 1627 (1989).

[9] M. Seul and M. J. Sammon, Thin Solid Films 185, 287 (1990).

[10] R. Podgornik and V. A. Parsegian, Biophys. J. 72, 942 (1997).

[11] C. R. Caillé, Acad. Sci. Ser. B 274, 891 (1972).

[12] L. Ning, C. R. Safinya, and R. Bruinsma, J. Phys. (Paris) II 5, 1155 (1995).

[13] L. Gunter, Y. Imry, and J. Lajzerowicz, Phys. Rev. A 22, 1733 (1980).

[14] J. Als-Nielsen et al., Phys. Rev. B 22, 312 (1980).

[15] C. R. Safinya, E. B. Sirota, D. Roux, and G. S. Smith, Phys. Rev. Lett. 62, 1134 (1989); 57, 2718 (1986).

[16] Note that the broad diffuse component is several orders of magnitude above the background, and is highly peaked in the radial $\left(q_{z}\right)$ direction. Furthermore, the diffuse scattering exhibits typical refraction effects [17], which would not be the case if the scattering would originate from badly oriented domains of the sample.

[17] S. K. Sinha, E. B. Sirota, S. Garoff, and H. B. Stanley, Phys. Rev. B 38, 2297 (1988).

[18] G. Niggemann, M. Kummrow, and W. Helfrich, J. Phys. (Paris) II 5, 413 (1995).

[19] The linear relationship should not be confused with the linearity of $\eta$ with absolute $T$ which is only a small effect within the measured range.

[20] A. Braslau, P. S. Pershan, G. Swislow, B. M. Ocko, and J. Als-Nielsen, Phys. Rev. A 38, 2457 (1988). 